\title{
2.3 Problematic issues of researching the structural increase in the reliability of engineering networks
}

In this monograph, the definition of engineering networks is presented, the formulation of the problem of maintaining their performance is considered. A large number of engineering networks functioning today are gradually reducing their capabilities as a result of the action of various destructive factors. Continuous maintenance and improvement of the level of performance of these systems is an important scientific problem. Therefore, the subject of this research is the process of geometric modeling of structural reliability on the example of redundant networks, which are one of the largest, most complex and important components of the engineering infrastructure.

Determining and ensuring reliability is an important problem in the design of water supply, heating, gas networks, networks of telecom operators, etc. Network-type structures are characteristic of many different extended geographically distributed systems. In a communication network, nodes are interconnected by channels that transmit flows of different origins: information (messages, data, control), energy (electrical or energy resources in the form of oil, gas or coal), material flows, transport. The structure of these systems and their functioning processes are quite similar, so their models can be same $[119,120,124]$.

Today, a complex of effective measures is needed to improve the recovery of industries and individual enterprises. At the same time, there are significant difficulties in building new and operational reconstruction of existing systems of engineering infrastructure and their components due to the difficult economic situation, inconsistency of plans and the availability of limited resources. A significant number of operating engineering networks were created long ago. And due to their long operating time, the stock and possibilities inherent in them are constantly decreasing. According to this, ensuring and increasing the level of reliability of engineering networks, increasing the period of their work is an important scientific problem [103, $110,111,120,126,127]$. This problem is being solved as a result of research and 
practical measures in order to weaken the action of various destructive factors that cause economic damage and other negative consequences [118].

The problem of the performance of different systems was investigated in scientific sources [103, 109, 113]. In the study of complex systems, various optimization methods are used [104, 105, 109, 113, 114, 121]. In particular, the issues of network optimization were worked out in the works [119]. The need for extended run times raises the requirements for system reliability and stimulates the expansion of the scope of their use. Loss of system reliability has great negative consequences [118].

Engineering and technical support networks (engineering networks, systems or communications) are a complex of systems and communications that ensure the vital activity of users (population, utilities and industrial enterprises) (Fig. 1). They are systems of communications and other structures, namely:

- external and internal water supply and drainage systems (water supply sources, hydraulic structures, water supply and sewage treatment plants, collectors, pumping stations, intra-quarter networks);

- external and internal heat supply systems (city heating networks, heating points and intra-quarter networks);

- gas supply systems (gas distribution points, gas pipelines, etc.);

- communication networks (cellular network, telephone network, structured cabling system, automated dispatch control system, access control system, visualization system);

- external and internal power supply systems (power lines, transformer and traction substations, etc.); internal power supply systems in houses;

- outdoor lighting systems (streets, roads, shop windows, stands, etc. and power lines);

- ventilation and air conditioning systems (residential and public buildings and structures and engineering facilities). 


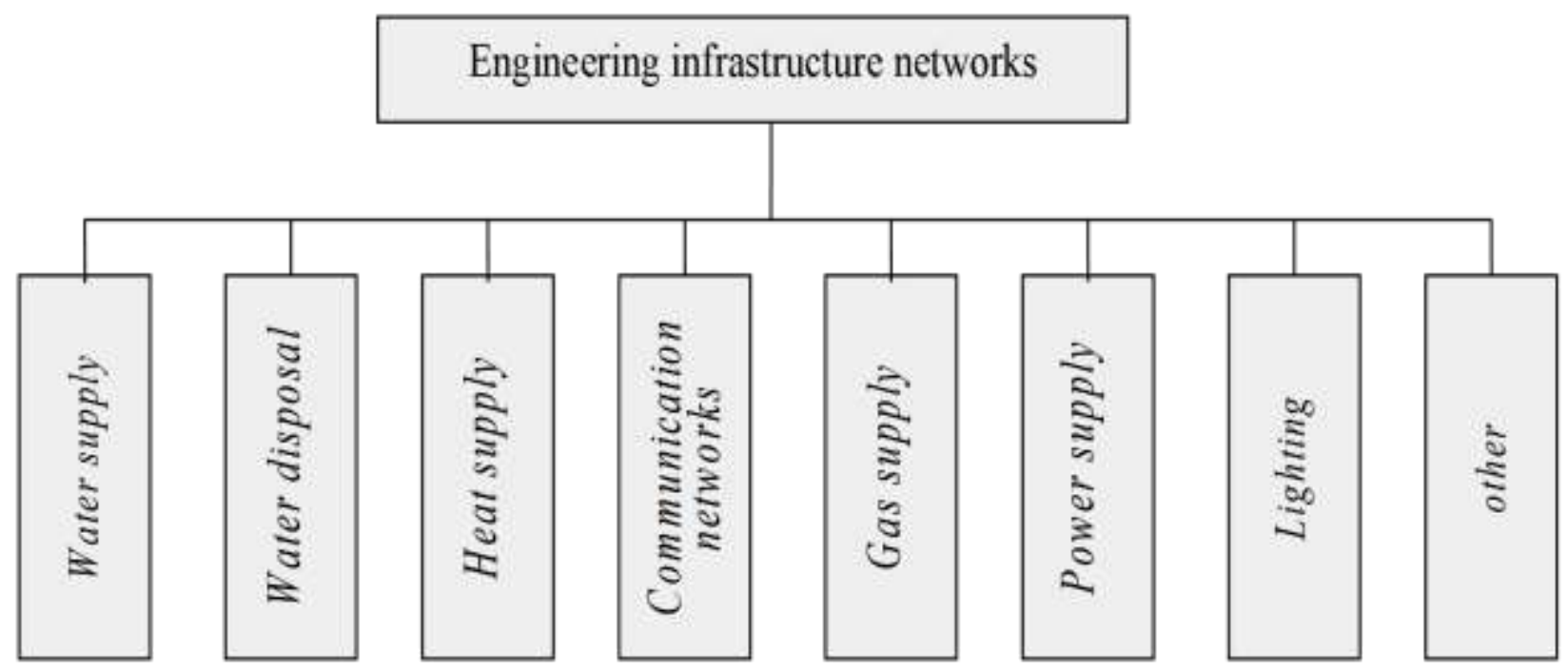

Figure 1. List of networks included in engineering infrastructure systems

Engineering networks are hierarchical and multilevel:

- they are perceived as a single complex object formed from interacting components of different levels, integrated to solve the assigned tasks with certain conditions:

- are systems of large dimension;

- losses from cessation of functioning are significant for society and the environment [103];

- significantly affect the economic situation and the efficiency of enterprises $[103,111]$.

The need to restore the utility network exists constantly for its functioning, the reliability of its elements is constantly decreasing and increasing, thereby affecting the operability of the system. An outstanding property of complex redundant engineering networks is the discrepancy between the volumes of losses from their serious failures and the costs of rational ensuring their performance [103, 111]. Maintaining a given level of reliable operation of these systems is critical, given certain limitations of the resources available for this. In the process of functioning, the object of the engineering infrastructure as a technical system performs its tasks of preserving a number of values of important quantities in the intervals specified in the regulatory documents [116]. To 
improve a certain level of reliability and upgrade complex systems, it is necessary to constantly invest large funds.

Reducing the technical capabilities of engineering networks and the need to continue their functioning is an urgent and difficult task to ensure their working condition. [103, 111]. The volume of an integrated study of this problem is insignificant, therefore, scientific support and finding hidden unused reserves is necessary. Structural redundancy uses additional components of the structure of the research object. According to [116]: redundancy is a method of ensuring system reliability by using additional capabilities that are more than the minimum necessary for the system to perform its tasks. The reserve element performs the functions of the main one in case of loss of its operability as part of a functioning system.

Modern objects of engineering infrastructure are characterized by high technical and functional-technological complexity, multi-level hierarchical and algorithmic functional link of constantly developing elements, improving in the process of adaptation to the environment. These objects are developing in several directions:

- more and more constituent elements are introduced into the systems;

- the structure of systems, formed by technological and functional connections of individual elements, becomes more complicated;

- different interactions of the components change during the functioning of the system. Today, engineering networks operate in conditions of the growing complexity of the tasks assigned to them, which are becoming more important, responsible and multivariate. Structural complexity, a number of increased requirements for performance, their numerous failures cause significant negative processes, significant losses of money and time. Research on these systems includes:

- finding a separate approach in their construction and analysis;

- consistency and application of methods for replacing or restoring components in the current state of the object in the process of their development;

- a variety of functions and scientific support for the entire period of existence.

- tasks of coordination, integration and interaction with subcontractors in the construction and operation of facilities; 
- limited amount of available resources for construction and operation.

Consequently, to maintain the performance of complex systems, it is necessary to improve, develop geometric methods, apply a systematic approach, methods of structural redundancy and geometric models to solve the assigned tasks.

Scientific literary sources indicate that the problem of reliability of complex network systems of engineering infrastructure is in the process of development and has not been finally solved $[103,111,117,120]$. Today, the general methodology for solving the problem of reliability of technical systems and their components of different levels of complexity is relevant. In particular, in scientific research, generalization and simplification of the system of connections of constituent parts in the structure of engineering networks is widely used. [103, 111, 117, 120]. Due to various problems of introducing and detailing theoretical methods, scientific sources $[103,111,117]$ became the basis for an in-depth analysis of the state of affairs for the further development of methods for modeling the operability of engineering networks. The study of the reliability of individual parts and elements of complex systems is carried out by methods of analyzing the reliability of operating objects and monitoring the operation of components of various types and dimensions as part of systems.

Under conditions of a high initial level of reliability of components or significant structural redundancy of engineering networks, the performance analysis leads to positive results, which indicates its low information content. In solving a number of practical problems, there is a need to determine and apply a number of transformations of the network structure that reduce the primary reliability of its components and elements in order to quickly obtain the necessary data on the level of performance and the use of inverse structural transformations. This helps to use the obtained data of computer experiments in calculating the actual indicators of the health of various components of the engineering infrastructure network.

In the practice of design, methods for calculating the performance indicators of engineering networks should be sufficiently accessible and not laborious $[103,111$, $117,120]$. The volume of calculations of the reliability of the object of analysis should not exceed the volume of other necessary engineering calculations associated with this 
system for sufficient practicality of the technique in the design process and in scientific support of the life of engineering networks.

Thus, the analysis of the state of the problem of the reliability of systems shows that for the development of research into the operability of engineering infrastructure networks, it is necessary to:

- construction of a generalized methodology for modeling their structural reliability and their various components;

- performing research on the reliability of individual components of network structures of different levels of complexity.

The development of science and technology and the preparation of a methodology for increasing and maintaining a given level of reliability of technical systems stimulate the deepening of theoretical methods of modeling reliability and methods of its practical calculations [129]. Scientific research methods are in constant development and open up broad prospects for solving a number of practical problems. Solving problems related to structural reliability uses graph analysis, checking them for the presence of different properties $[119,123]$. Graphs as mathematical models that arise in solving real practical problems are rather cumbersome and difficult in solving problems with a large dimension of network objects. Therefore, for efficient processing of these models, processing of the corresponding computational algorithms is required. $[119,123,125]$. Scientific research includes the processes of finding, selecting, creating and improving adequate models $[119,123]$. In these processes, important factors of influence on the object of research are selected for consideration and insignificant factors that are less significant for the identified circumstances and characteristics of the subject of research are rejected.

Reliability is considered to be a key property of all technical systems, regardless of their complexity. $[115,116]$. Without the required level of reliability, all other characteristics of the system have no practical meaning, because there is no possibility of their real application. [112, 115]. Therefore, reliability has an exceptional, leading role among all the properties of a technical system. [103, 107, 111, 112, 115, 119]. A number of qualitative characteristics of the subject of research have a significant impact 
on the level of its readiness for practical functioning in accordance with a given purpose. The study of the reliability of systems uses the means of mathematical statistics based on the results of experiments [106].

In the deterministic research approach, all the factors that determine the functioning of a certain system are taken completely unambiguous. Exceptionally accurate and specific data are used to consider the object of research with all its features. However, in practice, the process of functioning of various systems is stochastic and ambiguous [111, 112, 114, 119, 123, 125]. Therefore, the simulation results differ from the experimental data obtained under certain conditions [106]. Numerous system parameters are mostly of random origin. To model, determine and maintain the structural reliability of engineering infrastructure networks, it is necessary to take into account their constant changes during operation $[103,111,119,120,128$, 129]. Therefore, there are problems of finding the probabilistic properties of the processes in the system according to the given probabilistic properties of external conditions and in particular the geometric parameters of the system. This analysis is based on a scientific and technical problem, to solve which uses mathematical modeling, which should reflect the essence of this technical system.

In the scientific research of phenomena, objects and processes the description and forecasting of complex systems with the involvement of important probabilistic factors that constantly affect the course of their functioning is determined [108, 114]. Various relationships between stochastic laws and indicators of the level of system reliability are revealed. Experimental data are taken as probabilistic quantities varying according to some definite distribution law [106]. Of course, in describing the actions of the functioning of these engineering infrastructure systems, it is advisable to use a probabilistic analysis of their reliability.

The primary analysis of the reliability of the research object is carried out at the stage of system design. [103, 111]. The properties of the object's components are considered set. The study helps to remove unpromising solutions to the problem, identifies unreliable components of the research object and makes it possible to timely process all the "weak points" of the system. Further research of the system reliability 
is carried out according to the results of the statistical analysis of the experimental results obtained in the process of creating systems, their testing and operation. [106].

It is important to study the process of reducing the level of the primary parameters of the reliability of the system and its constituent parts. These changes depend on a number of existing patterns and are stochastic in nature, complex internal and external interactions and relationships. [114]. Interactions in the system are manifested through various characteristic manifestations of the existence of phenomena in the surrounding world. The accumulation of many significant influences stimulates changes in various parameters of the system's functioning in the direction of transition to another qualitative state. Therefore, changes occurring in the systems of engineering infrastructure in the course of their operation are a manifestation of many important properties of objects and surrounding processes.

Technical systems, in particular, engineering infrastructure facilities, at the beginning of work receive a certain maximum possible level of reliability under certain conditions. When performing the assigned tasks and under the influence of destructive factors, they lose the initial level of performance and go into a state of periodic failures. In general terms, the reliability of engineering infrastructure networks is determined by the probability of structural connectivity and random changes in the initial values of various parameters of the system within the limits corresponding to its performance. $[114,119,120,123]$. Therefore, the state of the system in one way or another depends on these fundamental parameters. In modeling the reliability of technical systems, research is constantly being conducted on the operability and reasons for the deterioration of the key properties of the system. The results of scientific developments are used to improve the theory, effectively organize experimental research (computer experiments), to develop means of regulating reliability and improve quality [106].

Based on the consideration of problematic issues, a generalized methodology for maintaining the operability of engineering infrastructure systems, in particular engineering networks, is drawn up, which is an integration of interrelated actions (stages) (Fig. 2). At the beginning of the study of the difficulty, a search is made for a method for analyzing and researching this system. In order to formalize dependencies, 
relationships, interactions and setting tasks in the process of performing a task, a system of geometric interpretations and ordering of initial data is built. In parallel, the formation (or improvement of the existing) object-oriented specialized terminology and notation system is underway, as well as the improvement of the system research method. The last step accompanies all stages of the research methodology.

Geometric modeling, analysis and synthesis of the research object are the next important stage in the search for a solution to the problem [129]. Here it is important to attract practical developments and generalize experience with a choice of methods for its study and the use of specialized terminology and a notation system. Obtaining the results of experiments, in particular computational ones, is necessary for their processing and appropriate interpretation. In order to accumulate, systematize and enrich scientific experience, theoretical descriptions and generalizations are carried out, solutions to the tasks and important conclusions are formulated.

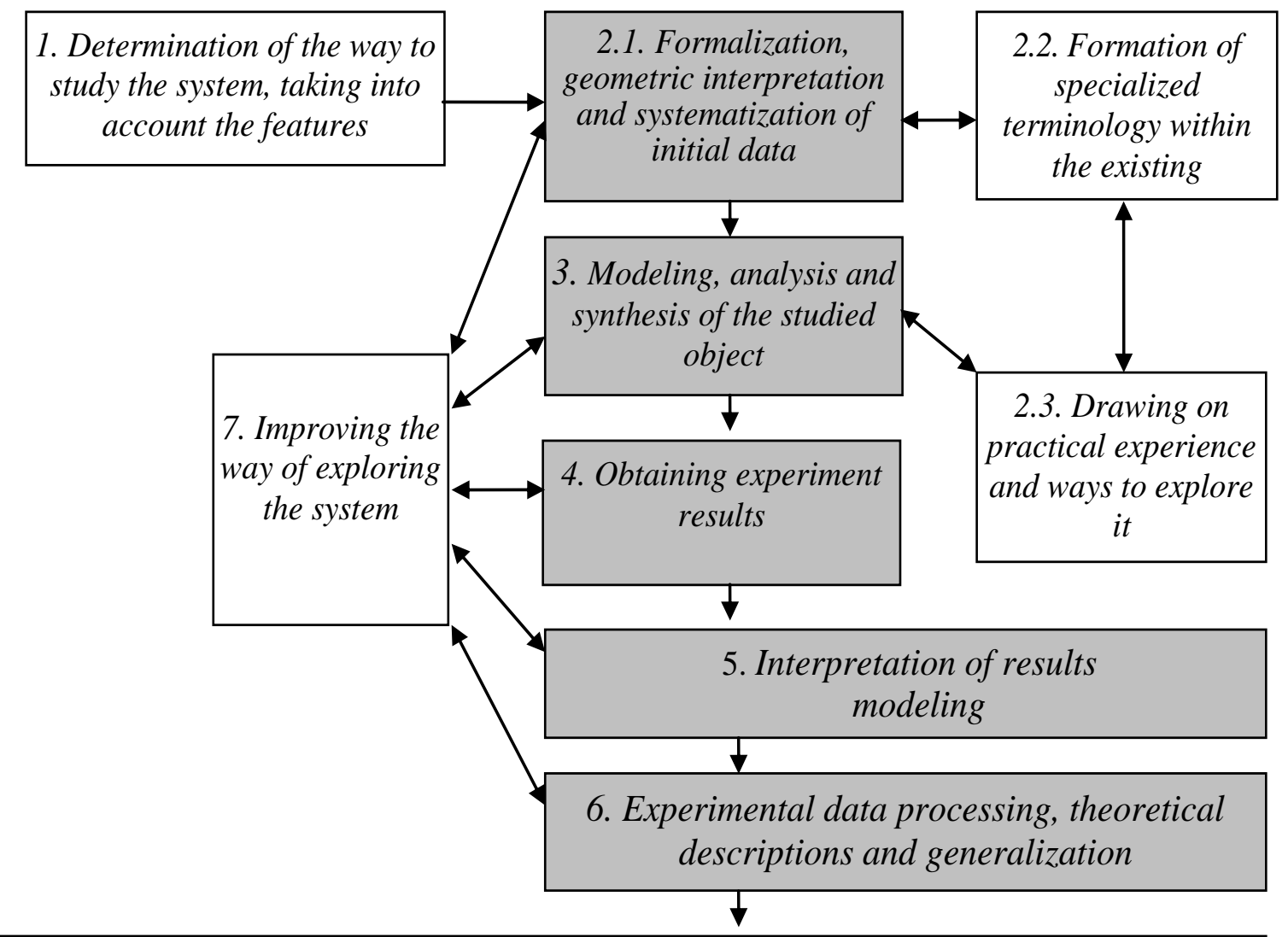

8. Formation of practical proposals and recommendations for solving the problem

Figure 2. Scheme of a generalized research methodology and ensuring the operability of engineering infrastructure systems from the standpoint of modeling 
The final stage of the methodology is the formation of a systematized series of proposals and recommendations for solving the problem of maintaining the operability of engineering infrastructure facilities. The generalized methodological structure of the theory of maintaining the operability of engineering infrastructure objects is based on geometric structural modeling [129]. The development of theoretical research in the field of applied geometry and engineering graphics is seen in the further formation and improvement of the system of their methods with the effective use of important factors influencing its important properties, in particular the reliability of various technical systems. The systems approach refines and integrates separate studies of these factors and properties. The methodological combination of a number of problems more adequately reflects the object and subject of research and expands the possibilities of geometric modeling and optimization. 